\title{
Expresión proteica del fluido folicular asociado a la calidad del oocito de vacas Cebú
}

\author{
Follicular fluid (FF) protein expression associated with oocyte quality \\ in Zebu cattle
}

\section{Expressão proteica do fluido folicular associado a qualidade do oócito de vacas Cebú}

\author{
Eliana Neira-Rivera ${ }^{1 *}$, Sonia L. Gutiérrez ${ }^{2 *}$, Lidy V. Castillo-Barón ${ }^{3 *}$, \\ José G. Velásquez-Penagos ${ }^{4 *}$, Agustín Góngora-Orjuela ${ }^{5 *}$; Jaime A. Cardozo-Cerquera ${ }^{6 *}$
}

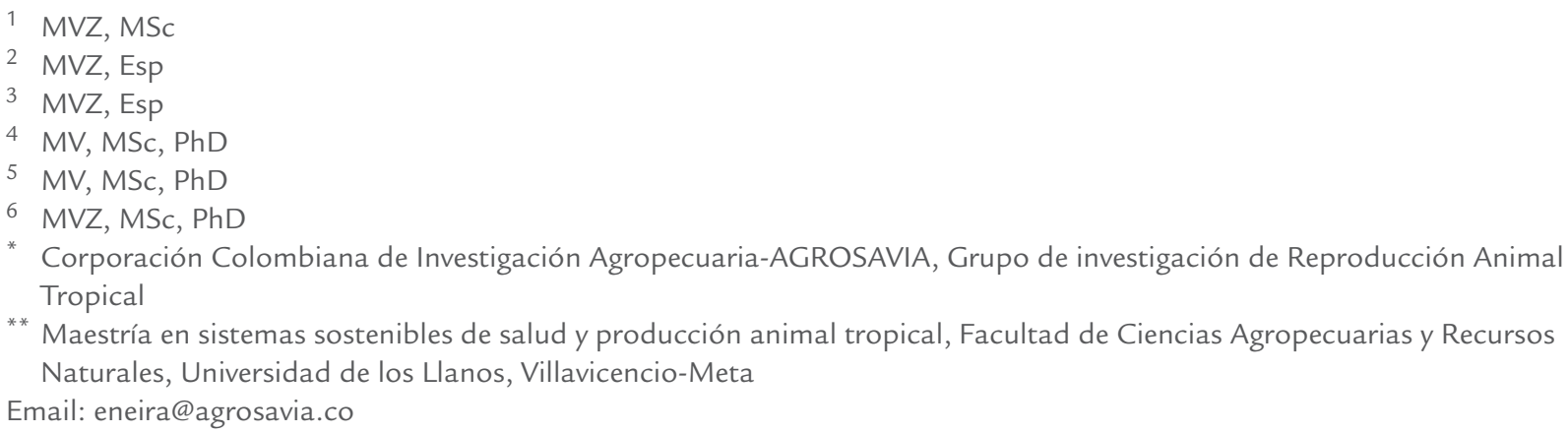

\section{Resumen}

El objetivo de este estudio fue determinar la expresión de proteínas del fluido folicular (FF) y su relación con la calidad del oocito. Se evaluaron 52 ovarios de planta de faenado de vacas Cebú comercial, mediante la técnica de disección y aspiración folicular se obtuvo FF y oocitos. Las evaluaciones realizadas fueron: calidad del oocito por aspecto citoplasmático y células del cúmulos y perfil de proteínas del FF mediante SDS-PAGE. Se realizó el análisis descriptivo, a través del procedimiento MEANS, análisis de varianza (PROC. ANOVA) y para las diferencias estadísticas significativas se usó la prueba de comparación de Bonferroni con un nivel de significancia del 5\%, mediante el paquete estadístico SAS®. El 52\% de los oocitos se categorizaron con calidad I-II. El análisis unidimensional de las proteínas del FF evidenció la presencia de 25 bandas de proteína entre 9 y $240 \mathrm{kDa}$. En folículos $<3 \mathrm{~mm}$ se expresaron 23 bandas, en folículos de 3 y $6 \mathrm{~mm} 19$ bandas y en folículos $>6 \mathrm{~mm} 20$ bandas. Las bandas de peso molecular (PM) de 26kDa, 57kDa y 68kDa representan la mayor concentración en el FF; 4 bandas de PM 14 KDa, 34 KDa, 76 y 79 KDa, solo en folículos de <3mm, 2 bandas de PM 9 y 91 KDa 
solo en folículos de $>3 \mathrm{~mm}$. La banda de $32 \mathrm{KDa}$ no se observó en folículos $>$ de $6 \mathrm{~mm}$. Las bandas de mayor frecuencia de presentación fueron las de 26, 40, 42, 57, 68, $240 \mathrm{KDa}$. Las bandas de proteína que se asociaron con la calidad del oocito en forma significativa $(p<0,05)$ fueron las de PM 24, 57, 68 y 164 KDa para FF de folículos <3mm y las bandas de PM 13, 26 y $38 \mathrm{kDa}$ entre 3 y $6 \mathrm{~mm}$, y la de $26 \mathrm{kDa}$ a folículos > de $6 \mathrm{~mm}$. Los resultados nos indican asociaciones de la calidad del oocito con algunas bandas de proteína.

Palabras clave: Folículo bovino; bandas de proteínas; oocito; perfiles proteícos

\begin{abstract}
This study was aimed at determining follicular fluid (FF) protein expression and its relationship with oocyte quality. FF and oocytes were obtained by dissection and follicular aspiration of the ovaries from fifty-two commercial Zebu from a slaughterhouse. Oocyte quality was measured by cytoplasmic aspect and cumulus cells and FF protein profile by SDS-PAGE. The SAS statistical package's PROC MEANS and analysis of variance (ANOVA) were used for descriptive analysis and the Bonferroni comparison test for assessing significant statistical differences (5\% significance level); $52 \%$ of the oocytes were categorised as having I-II quality. One-dimensional SDS-PAGE analysis of FF proteins revealed 25 protein bands having 9 $\mathrm{kDa}$ to $240 \mathrm{kDa}$ molecular weight (MW); 23 bands were expressed in $<3 \mathrm{~mm}$ follicles, 19 bands in 3 and $6 \mathrm{~mm}$ follicles and 20 bands in $>6 \mathrm{~mm}$ follicles. The $26 \mathrm{kDa}, 57 \mathrm{kDa}$ and $68 \mathrm{kDa}$ bands' $\mathrm{MW}$ represented the highest FF concentration whereas only four bands (14 kDa, $34 \mathrm{kDa}, 76$ and $79 \mathrm{kDa} M W$ ) were found in <3mm follicles and only 2 bands (9kDa and $91 \mathrm{kDa}$ MW) in $>3 \mathrm{~mm}$ follicles. The $32 \mathrm{kDa}$ band was not observed in $>6 \mathrm{~mm}$ follicles. The 26, 40, 42, 57, 68 and $240 \mathrm{KDa}$ bands occurred with the greatest frequency. The protein bands which were significantly associated with oocyte quality $(p<0.05)$ were 24, 57, 68 and $164 \mathrm{kDa}$ MW for <3mm follicles, 13, 26 and $38 \mathrm{kDa}$ MW bands for 3 and $6 \mathrm{~mm}$ follicles and $26 \mathrm{kDa}$ for $>6 \mathrm{~mm}$ follicles. The results indicated oocyte quality association with some protein bands.
\end{abstract}

Key words: bovine follicle; protein band; oocyte; protein profile.

\title{
Resumo
}

Este estudo teve como objetivo determinar a expressão de proteínas do fluido folicular (FF) e sua relação com a qualidade do oócito. Avaliou-se 52 ovários de vacas Cebú comercial colhidos em abatedouro e através da técnica de dissecação e aspiração folicular obtivera-se o FF e os oócitos. As avaliações realizadas foram: qualidade do oócito pelo aspecto citoplasmático e células do cúmulos e o perfil de proteínas do FF mediante SDS-PAGE. Realizou-se a análise descritiva, através do procedimento MEANS, análise de variância (PROC. ANOVA) e para identificar as diferenças estatísticas significativas, utilizouse a prova de comparação de Bonferroni com um nível de significância de 5\%, através do pacote estadístico SAS®. O $52 \%$ dos oócitos foram classificados como qualidade I e II. As análises unidimensionais das proteínas do FF evidenciaram a presença de 25 bandas de proteína entre 9 y $240 \mathrm{kDa}$. Em folículos <3 mm expressaram-se 23 bandas, em folículos entre 3 e 6 mm 19 bandas e em folículos >6mm 20 bandas. As bandas de peso molecular (PM) de 26kDa, 57kDa e 68kDa são as mais frequentes no FF; 4 bandas de PM $14 \mathrm{KDa}, 34 \mathrm{KDa}, 76$ y $79 \mathrm{KDa}$, solo em folículos de <3mm, 2 bandas de PM 9 y $91 \mathrm{KDa}$ solo em folículos de $>3 \mathrm{~mm}$. A banda de $32 \mathrm{KDa}$ não foi observada em folículos $>$ de $6 \mathrm{~mm}$. As bandas mais frequentes foram 26, 40, 42, 57, 68, $240 \mathrm{KDa}$. As bandas de proteína que se associaram à qualidade do oócito em forma significativa $(p<0,05)$ foram as de PM 24, 57, 68 e 164 KDa para FF de folículos <3mm e as bandas de PM 13, 26 e 38 kDa entre 3 e $6 \mathrm{~mm}$, e a de $26 \mathrm{kDa}$ a folículos $>6 \mathrm{~mm}$. Os resultados indicam associações entre qualidade do oócito e algumas bandas de proteína.

Palavras-chave: Folículo bovino; fluido folicular; oócito; bandas de proteína

\section{Introducción}

Los procesos fisiológicos relacionados con el crecimiento y degeneración de los folículos ováricos durante el ciclo estral en bovinos han sido tema de los grupos de investigación durante las últimas décadas. El desarrollo folicular es un proceso dinámico caracterizado por la proliferación y diferenciación celular, cuyo fin es proporcionar un medioambiente óptimo para la maduración del oocito y posterior preparación para su fecundación (Amstrong y Webb, 1996). El entendimiento de la dinámica y el proceso de maduración requiere del conocimiento de los diversos aspectos que lo influencian como la esteroidogénesis, la parti- cipación de sustancias en el fluido folicular (FF) que intervienen en la diferenciación celular, el crecimiento del folículo y la ovulación (Petrucci et al., 2014), así como su efecto en las diversas formas de competencia adquiridas por el oocito. Así mismo los estudios de la composición del FF han llegado a generar indicaciones de requerimientos celulares que pueden ser usados como una guía en la formulación de medios de cultivo celular en procesos de producción de embriones in vitro (Gerard et al., 2002).

El FF contiene proteínas entre otras moléculas que inciden en los procesos de maduración nuclear y citoplasmática del oocito (Sirard et al., 1998), es especí- 
fico de cada especie y puede presentar variaciones en su composición, dependientes del estado fisiológico. Dentro los procesos de maduración oocitaria se encuentran modificaciones moleculares (Kubelka et al., 2000), redistribución de organelos intracelulares (Stojkovic et al., 2001) y los mecanismos de liberación de Ca2+ (Wang et al., 2003).

Los cambios estructurales en el oocito están acompañados de una serie de procesos bioquímicos, que establecen una compleja cascada de eventos de fosforilación y desfosforilación de proteínas, que involucran el reinicio y la regulación de la meiosis (Sousa et al., 2004, Dekel 2005 y Dumont et al., 2005). La pérdida de sincronía de este tipo de eventos va a influir negativamente en el desempeño y calidad de los oocitos que originaría una baja de producción de embriones viables.

Los estudios adelantados en Colombia sobre dinámica folicular han empleado herramientas como el ultrasonido y la determinación de perfiles bioquímicos en sangre. Como se mencionó anteriormente la determinación de proteínas en el FF proporcionan una comprensión más profunda en los procesos de desarrollo folicular y maduración oocitaria por la cual el objetivo de este estudio fue determinar los cambios en los perfiles de las proteínas del fluido folicular relacionados con la calidad del oocito.

\section{Materiales y métodos}

En este estudio se utilizaron ovarios, folículos, FF y oocitos de tractos uterinos de vacas Cebú comercial obtenidos en el frigorífíco-Friogan de Villavicencio, Meta. La recolección del FF, y la evaluación de folículos y oocitos y la cuantificación y separación electroforética de proteínas del fluido folicular se llevó a cabo en la Corporación Colombiana de Investigación Agropecuaria (AGROSAVIA), en el Laboratorio de Reproducción del centro de Investigación $(\mathrm{Cl}) \mathrm{La} \mathrm{Li}$ bertad, ubicado en el kilómetro 17 vía Puerto López, departamento del Meta a una altura de $467 \mathrm{mts}$ sobre el nivel del mar y en el laboratorio de Proteómica del centro de Investigación $(\mathrm{Cl})$ Tibaitatá, ubicado en el kilómetro 14 vía Mosquera, del departamento de Cundinamarca.

\section{Obtención de los Ovarios}

Los ovarios fueron extraídos de tractos uterinos de 26 hembras bovinas cíclicas (presencia de cuerpo lúteo y folículos en el ovario), de la raza cebú comercial adultas. Una vez extraídos los tractos uterinos del animal, se depositaron en una bolsa de colecta, con so- lución de tampón fosfato salino estéril (PBS) (por sus siglas en inglés), o con solución salina $0.9 \%$ estéril a $36 \pm 1{ }^{\circ} \mathrm{C} \%$.

\section{Evaluación del fluido folicular}

El fluido folicular se obtuvo por aspiración directa del folículo ovárico con aguja calibre 16 acoplada a una jeringa de $10 \mathrm{~mL}$, su contenido se colocó en viales de $2 \mathrm{~mL}$; posteriormente se realizó la búsqueda de oocitos y separación del fluido folicular.

La muestra de fluido folicular se centrifugó a 13.000 rpm durante 30 minutos a $4^{\circ} \mathrm{C}$, posteriormente se adicionó inhibidor de proteasas (fenilmetilsulfonil fluoruro) disuelto en dimetil sulfoxido (DMSO) (por sus siglas en inglés), $3 \mu \mathrm{l}$ por cada $1 \mathrm{ml}$ de muestra, y se almacenó a $-20{ }^{\circ} \mathrm{C}$ para su sucesivo uso.

Las proteínas se cuantificaron mediante el método colorimétrico descrito por Bradford, 1976. La separación de las proteínas de fluido folicular se realizó mediante electroforesis 1D SDS-PAGE de tipo desnaturalizante y discontinuo, según protocolo de Laemmli (1970). Los geles se digitalizaron en el documentador de imágenes Molecular Imagen Gel Doc, de Bio-Rad®. El análisis de los geles se realizó con el software Quantity One ${ }^{\circledR}$ de Bio-Rad.

\section{Extracción de folículos y clasificación}

Luego de la recuperación de los ovarios, se extrajeron los folículos, estos fueron puestos en una placa de petri con solución de tampón fosfato salino estéril (PBS) sobre una platina calentadora $36^{\circ} \mathrm{C}$; siguiendo la técnica de Caixeta et al., 2008.

Posterior a la disección de los folículos, la medición del tamaño se realizó con ayuda de una lupa con ocular graduado. (Estereoscopio, Olympus ${ }^{\circledR}$ ). Los folículos se categorizaron según su tamaño así: Folículos con un tamaño entre 1 a 3,0 mm (TI), folículos con un tamaño entre 3,1 a $6 \mathrm{~mm}$ (TII) y folículos con un tamaño 6,1 a $8 \mathrm{~mm}$ (TIII).

\section{Evaluación de la calidad oocito}

La evaluación de la calidad del oocito se realizó con base en el aspecto de su citoplasma y las características del cúmulo que lo rodea, para ello nos apoyamos en lo descrito por Solís et al., 2012 y la modificación que se consideró fue la integración del grado III y IV. Evaluando en este estudio los grados I (GI), II (GII) y III (GIII) (tabla 1). 
Tabla 1. Criterios de selección de los COCs Fuente: Solís et al., 2012.

\begin{tabular}{|l|l|}
\hline Clasificación & \multicolumn{1}{c|}{ Descripción } \\
\hline Grado I & $\begin{array}{l}\text { Cúmulos con capas múltiples, } \\
\text { Cúmulos compacto, La totalidad } \\
\text { del cúmulos es clara y transparente, } \\
\text { citoplasma homogéneo }\end{array}$ \\
\hline Grado II & $\begin{array}{l}\text { Cúmulos como grado uno o algo } \\
\text { más oscuro y menos y transparente, } \\
\text { Citoplasma con granulación } \\
\text { más gruesa y más oscura en la } \\
\text { periferia que en grado uno }\end{array}$ \\
\hline Grado III & $\begin{array}{l}\text { Cúmulos menos compacto, } \\
\text { más oscuro que en uno o dos, } \\
\text { Citoplasma con manchas oscuras }\end{array}$ \\
\hline Grado IV & Sin cúmulos o expandidos \\
\hline
\end{tabular}

\section{Análisis estadístico}

El análisis de la información se apoyó en estadística descriptiva, utilizando el paquete estadístico $S A S \circledast$, procedimiento MEANS, ANAVA, y CORR. Para las diferencias estadísticas significativas se usó la prueba de comparación de Bonferroni con un nivel de significancia del $5 \%$.

\section{Resultados}

\section{Proteínas totales del FF en folículos de ovarios de bovinos}

La concentración promedio de proteína total del FF en

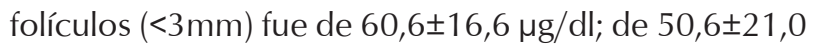
$\mu \mathrm{g} / \mathrm{dl}$ en folículos $(3-6 \mathrm{~mm}$ ) y de $53,4 \pm 16,3 \mu \mathrm{g} / \mathrm{dl}$ en folículos mayores de $6 \mathrm{~mm}$. Al análisis estadístico, estos valores no presentan diferencias $(p>0,05)$ en función del tamaño del folículo.

En el fluido de los folículos de 3-6 mm la concentración de proteína total correlacionó positivamente $(r=0,29$, $p<0,05)$ con oocitos de buena calidad, $y$ en oocitos de mala calidad $(r=0,27, p<0,05)$. En folículos $>6 \mathrm{~mm}$ la concentración de proteína total correlacionó $(r=0,39$, $p<0,05)$ con oocitos Gll.

En estos resultados, se pudo detectar una correlación $(r=0,43, p<0,05)$ para la concentración de proteína de fluido folicular de folículos $>6 \mathrm{~mm}$ y oocitos GIl en el lado derecho del ovario.
En el ovario izquierdo la concentración de proteína del fluido folicular de folículos de 3-6 mm correlacionó $(r=0,37, p<0,05)$ con oocitos Gll y $(r=0,39, p<0,05)$ con oocitos GIII. Mientras que en el fluido de los folículos $>6$ mm se encontró una correlación $(r=0,40, p<0,05)$ con la calidad oocito GII.

\section{Perfiles electroforéticos unidimensionales SDS- PAGE de proteínas del FF en folículos de diferentes tamaños}

El análisis de las imágenes de los geles unidimensionales permitió establecer a nivel general, un número de 25 bandas de proteína expresadas en el FF de los diferentes folículos evaluados, con pesos moleculares entre 9 y 240 kDa (figura 1). En folículos de tamaño I se expresaron un total de 23 bandas de proteína con pesos moleculares entre 13 a $240 \mathrm{kDa}, 19$ bandas de proteína entre 9 a $240 \mathrm{kDa}$ se expresaron en FF de folículos de tamaño II, y 20 bandas de proteína entre 9 y 240 kDa se expresaron en FF de folículos con tamaño III.

Las proteínas de 14 kDa, 34 kDa, 76 kDa y 79 kDa únicamente se expresaron en el fluido folicular de folículos de tamaño I $(<3 \mathrm{~mm})$, de igual forma la banda de proteína de $32 \mathrm{kDa}$ se presentó en los folículos de tamaños I y II y las bandas de proteína de 24 y 98 kDa en los folículos de tamaño I y III, por otra parte las bandas de proteína de 9 y 91 kDa no se presentaron en los folículos de tamaño I (figura 2).

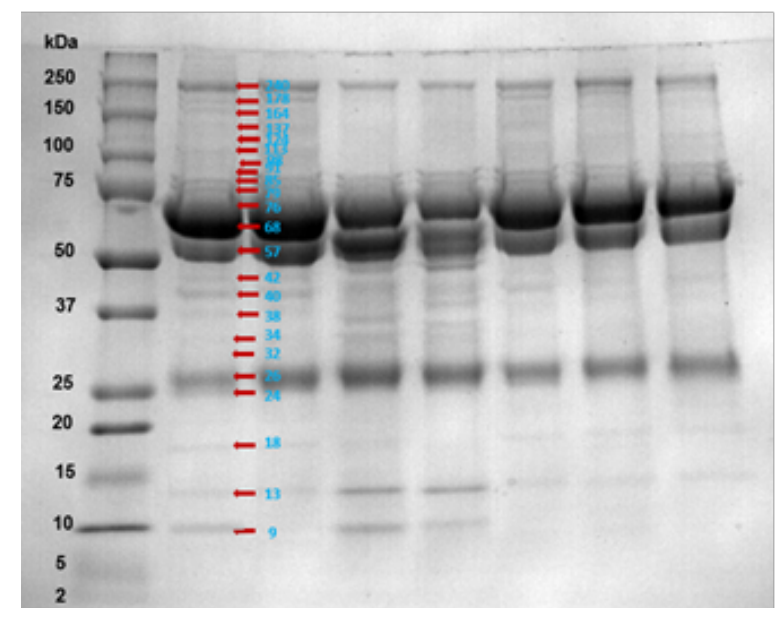

Figura 1. Perfil electroforético unidimensional de bandas de proteína de fluido folicular de ovarios de vacas Cebú comercial. El marcador de peso molecular, se ubicó al lado izquierdo de la imagen, el peso molecular de las bandas de proteínas se relacionaron en kilo Dalton $(\mathrm{kDa})$. Con flechas café se señalan las bandas de proteínas presentes en este estudio. 


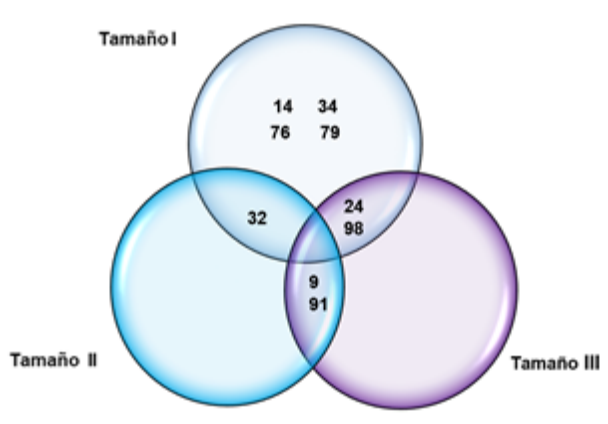

Figura 2. Análisis de expresión de bandas de proteínas de fluido folicular (peso molecular) presentes en el perfil electroforético en cada tamaño de folículo.

\section{Concentración de las bandas de proteína de fluido folicular en folículos de tamaño I, II y III}

La banda de proteína con peso molecular de $68 \mathrm{kDa}$, representa el $24,45 \%$ del total de la cantidad relativa de proteína del FF, la banda de 57 kDa el 13,65\%, y la banda de $26 \mathrm{kDa}$ el $20,81 \%$, estas bandas son las que identifican a un grupo de proteínas del FF con un promedio de concentración relativo mayor, que constituyen el $58,91 \%$ de la concentración total. En contraste las bandas de proteínas de 210,164,98,91,79,76,34,32,24,14 y $9 \mathrm{kDa}$, representan solo el $7,38 \%$. Sobresalen en los resultados, la banda de $68 \mathrm{kDa}$ como la de mayor concentración en el FF de los tres tamaños de folículo, seguida de la banda de $26 \mathrm{kDa}$ y de $57 \mathrm{kDa}$. Es notorio que a medida que aumenta el tamaño folicular, aumenta la concentración de las bandas de 68 y 57 kDa, mientras es estable la concentración para la banda de $26 \mathrm{kDa}$. Se evidenció diferencia estadística $(p<0,05)$ en la concentración relativa de la banda de $164 \mathrm{kDa}$, entre el ovario derecho e izquierdo.

\section{Frecuencia de presentación de las bandas de proteína de fluido folicular en folículos de tamaño I, II y III}

Las bandas de proteínas con mayor frecuencia de presentación $(80 \%)$ en folículos de tamaño I fueron las de $240 \mathrm{kDa}, 68 \mathrm{kDa}, 57 \mathrm{kDa}, 26 \mathrm{kDa}, 40$ y $42 \mathrm{kDa}$ y las bandas de 34, 24 y $14 \mathrm{kDa}$ fueron las de menor frecuencia (20\%). En folículos de tamaño II, las bandas de $240 \mathrm{kDa}, 57 \mathrm{kDa}, 26 \mathrm{kDa}, 68 \mathrm{kDa}, 85 \mathrm{kDa}, 40$ $\mathrm{kDa}, 137 \mathrm{kDa}, 178 \mathrm{kDa}, 42 \mathrm{kDa}$ fueron las de mayor frecuencia y las de menor presentación fueron las bandas de $32 \mathrm{kDa}, 9 \mathrm{kDa}, 210 \mathrm{kDa}, 91 \mathrm{kDa}$, y finalmente en folículos de tamaño III, las bandas por encima del $80 \%$ de presentación son las de $240 \mathrm{kDa}, 210 \mathrm{kDa}, 68$ kDa, 57 kDa , 26 kDa, 85 kDa ,137 kDa, 40 kDa, y con menor presentación las de 9 kDa, 42 kDa, 91 kDa, 124 kDa, 178 kDa, 24 kDa, 13 kDa, y 18 kDa.

\section{Relación entre las bandas de proteínas del Fluido Folicular provenientes de folículos de diferentes tamaños y la calidad del oocito}

Se observó correlación $(r=0.66, p<0.001)$ entre la banda de 164 kDa y la calidad II del oocito, para el tamaño I del folículo. En este mismo tamaño de folículo se encontró que la proteína de 57 kDa se correlacionó inversamente $(r=-0.36 ; p<0,05)$ con el oocito de calidad III. En el FF del folículo de tamaño II, la proteína de $38 \mathrm{kDa}$ se correlacionó $(\mathrm{r}=0.68, \mathrm{p}<0.003)$ con la calidad I del oocito, en tanto que la banda de $26 \mathrm{kDa}$ correlacionó $(r=-0.42, p<0.02)$ con oocitos de este mismo grado de calidad. En el tamaño III del folículo, la banda de 26 kDa correlacionó ( $r=-0.53$, p<0.008) con oocitos de calidad Gll.

\section{Asociación entre las proteínas del FF y la calidad del oocito en folículos de diferente tamaño en el ovario derecho}

En fluido de folículos de tamaño I, la banda de 164 kDa correlacionó ( $r=0.8, p<0,05$ con oocitos calidad $\mathrm{Gl}$, mientras que oocitos de calidad GIII correlacionaron con las bandas de $68 \mathrm{KDa}, 57 \mathrm{KDa}(\mathrm{r}=-0.45 \mathrm{y}$ -0.62 respectivamente, $\mathrm{p}<0.05)$. En fluido de folículos de tamaño II, las bandas de $38 \mathrm{KDa}$ y $26 \mathrm{KDa}$ correlacionaron ( $r=0.74$ y -0.58 respectivamente, $p<0.05$ ) con oocitos calidad Gl. La calidad Gll del oocito correlacionó $(r=0.72, p<0.05)$ con la banda de 13KDa. Las bandas de proteína de FF de folículos tamaño III, no presentaron correlaciones con la calidad del oocito del ovario derecho.

\section{Asociación entre las proteínas del FF y la calidad del oocito en folículos de diferente tamaño en el ovario izquierdo}

En fluido de folículos de tamaño I del folículo, la banda $164 \mathrm{kDa}$ correlacionaron $(r=0,62, \mathrm{p}<0,05)$ con oocitos de buena calidad. La calidad Gll del oocito, correlacionó $(r=0.82, p<0.02)$ con la banda de $98 \mathrm{KDa}$, mientras que el oocito calidad GIll correlacionó $(r=0.82$, $\mathrm{p}<0.023$ ) con la banda de $210 \mathrm{KDa}$. En el fluido del folículo de tamaño II, la banda de 124 kDa correlacionó $(r=0.89, p<0.05)$ con el grado de calidad GIII del oocito, mientras que la banda de $68 \mathrm{KDa}$ correlacionó $(r=0.74, p<0.05)$. Finalmente, en el fluido del folículo tamaño III, las bandas de $68 \mathrm{kDa}$ y $26 \mathrm{KDa}$ se correlacionaron ( $r=0.71$ y -0.63 respectivamente, $p<0.05)$ con calidad del oocito grado II. 


\section{Calidad del oocito}

Los 403 oocitos evaluados en esta investigación fueron colectadas 321 en folículos tamaño I, 69 folículos tamaño II y 13 folículos tamaño III. Se clasificaron en calidad Gl (93), Gll (115) y GIII (195) oocitos. Para el tamaño de los folículos $<3 \mathrm{~mm}$ los oocitos fueron de calidad Gl: 73, Gll:52 y GIII: 158, en el tamaño II en calidad GI:20, GII:54, y en GIII:33 y finalmente en folículos definidos como mayores de $6 \mathrm{~mm}$ en calidad GII: 9 y GIII:4. En la tabla 2, se observa los promedios de oocitos obtenidos de folículos aislados del ovario derecho e izquierdo colectados de estructuras de matadero por grado de calidad (G I, GII, GII) clasificados por la escala de Solís et al., 2012, los resultados indican que para todos los tamaños determinados I, II, y III, en los grados de calidad (GI, GII,GIII) únicamente se encontró diferencia entre los oocitos de folículos de ovario derecho e izquierdo de tamaño II (3-6 mm) y clasificación de calidad GIII (Cúmulos como grado uno o algo más oscuro y menos y transparente, citoplasma con granulación más gruesa y más oscura en la periferia que en grado uno) en forma significativa $(p<0.05)$.

\section{Discusión}

Las concentraciones de proteínas de fluido folicular de esta investigación se encuentran dentro de los rangos promedio de concentración de proteínas totales en fluido folicular (Villa et al., 2009; Nandi et al., 2007; Leroy et al., 2004); sin embargo, nuestros resultados difieren de los de Aller et al., 2013; Shabankareh et al., 2013 en bovinos, que describen una disminución en la concentración total de proteínas a medida que aumentaba el tamaño del folículo. Aspectos como el diámetro folicular, crecimiento oocitario y folicular conllevan a fluctuaciones en la concentración de las proteínas y otros metabolitos por una mayor capacidad de filtración en la estructura folicular en folículos grandes (Fortune y Hansel, 1985; Nandi et al., 2007; Rahman et al., 2008; Tabatabaei et al., 2011); y también es mayor en folículos sanos (folículos irrigados) a diferencia de folículos atresicos según Clarke et al., 2006. Lo anterior respalda los resultados encontrados en esta investigación.

Para determinar la presencia de bandas de proteínas del fluido de folículos las muestras se sometieron a SDS-PAGE, evidenciándose un número de 25 bandas de proteínas similares a los descritos por Ribeiro et al., 2012 e inferiores a los encontrados por Mortarino et al.,1999 y Zachut et al.,2016; y a los encontrados por Petrucci et al., 2014 en folículos entre 15 y 40 mm; y en otros estudios de proteómica realizados en fluido folicular en búfalos (Fu et al., 2016). Las bandas de proteínas detectadas en fluido folicular bovino denotan la presencia de albuminas, cadenas pesadas de inmunoglobulinas, alfa-1-antitripsina, alfa-2-macroglobulinas, angiotensinas, apolipoproteinas, antitrombinas, fibrinógeno, haptoglobulinas, lactoferrinas, transferrinas entre otras (Sunderland et al., 1996; Driancourt, 2001; Fahiminiya et al., 2011; Ambekar et al., 2013).

Como se expresó anteriormente las bandas de proteínas con pesos moleculares de 14 y $34 \mathrm{kDa}$ solo se detectaron en folículos menores $3 \mathrm{~mm}$, su peso molecular sugiere que se trate de las haptoglobulinas $\alpha$

Tabla 2. Promedios de oocitos clasificados por tamaño y calidad obtenidos de folículos de ovarios derecho e izquierdo.

\begin{tabular}{|c|c|c|c|}
\hline Tamaño & Calidad & $\begin{array}{l}\text { Oocitos de folículos } \\
\text { del ovario derecho }\end{array}$ & $\begin{array}{l}\text { Oocitos de folículos } \\
\text { del ovario izquierdo }\end{array}$ \\
\hline \multirow{3}{*}{ I $(<3$ mm) } & G I & $1,42 \pm 2,21 \mathrm{a}$ & $1,38 \pm 1,79 a$ \\
\hline & GII & $2,15 \pm 2,37 a$ & $1,30 \pm 1,56 a$ \\
\hline & GIII & $2,80 \pm 2,38 \mathrm{a}$ & $3,26 \pm 3,40 \mathrm{a}$ \\
\hline \multirow{3}{*}{ II (3-6 mm) } & G I & $0,61 \pm 1,16 a$ & $0,15 \pm 0,36 a$ \\
\hline & GII & $0,42 \pm 0,75 a$ & $0,19 \pm 0,49 a$ \\
\hline & GIII* & $0,96 \pm 1,45 a$ & $0,30 \pm 0,78 b$ \\
\hline \multirow{3}{*}{ III (>6mm) } & G I & - & - \\
\hline & GII & $0,11 \pm 0,32 \mathrm{a}$ & $0,23 \pm 0,65^{\underline{a}}$ \\
\hline & GIII & $0,13 \pm 0,196 a$ & $0,11 \pm 0,32^{\underline{a}}$ \\
\hline
\end{tabular}

Media \pm SEM; medias con letras diferentes indican diferencias entre grupos de tipo significativo ${ }^{*} p<0,05$ o altamente significativo $* * 0<0.001$ 
1 chain; factores de crecimiento entre ellos IGFB2, caracterizados por aumentar la proliferación y diferenciación de células foliculares según Monget et al., 1996; inmunoglobulinas con acciones en el espermatozoide y la interacción de este con el oocito, y síntesis en respuesta a agentes infecciosos (Fahiminiya et al., 2011). Así mismo en este tamaño se encontraron las bandas de 76 y $79 \mathrm{kDa}$ que pueden corresponder con la familia de las transferrinas (de Jong et al.,1990) involucradas en el transporte de hierro, la reducción de ROS y procesos de atresia folicular. La banda de proteína de $32 \mathrm{kDa}$ se identificó en los folículos de tamaño I y II, que puede corresponder a los factores de complemento C3 (31,84 PM aprox.) según Bianchi et al., 2013; los genes de esta proteína tienen acción reguladora de hormonas tiroideas, glucocorticoides y de estrógenos entre otros (Fahiminiya et al., 2010; Yoo et al., 2013). Las bandas 98 y 24 kDa se encontraron en el fluido de folículos de tamaño I y III, la primera puede ser una proteína de la familia de las gelatinasas, y la segunda corresponder a factores de crecimiento insulínico (tipo 4) de importancia en procesos de crecimiento y desarrollo folicular. Las bandas de 9 y 91 kDa detectadas en los tamaños II y III, podrían ser: la de 9 kDa una proteína relacionada con el factor de crecimiento como el epidermal, este tipo de proteínas son exclusivas de la interacción de las células granulosa, de la teca y el metabolismo folicular (Schweigert et al., 2006, Hill 1989 y Jans et al., 1998). La banda de proteína de $91 \mathrm{kDa}$ identificada podría ser una proteína relacionada con la gelsolina, participa en procesos contráctiles y morfogenéticos que ocurren durante el desarrollo folicular, ovulación modulando la actividad de la actomisina ATPasa (Teubner et al., 1994).

En este estudio se encontró que las bandas $68 \mathrm{kDa}$, $57 \mathrm{kDa}, 26 \mathrm{kDa}$ son las que representan mayor concentración en los tres tamaños foliculares. Ribeiro et al., 2012 y Gradela et al., 1998 reportaron como bandas de mayor concentración las de 68 y 26 kDa. La concentración de las bandas de proteína de 68 y 57 $\mathrm{kDa}$ aumenta en forma progresiva a medida que el tamaño folicular es mayor. La banda de $68 \mathrm{KDa}$ podría corresponder a la albumina sérica bovina que fuera reportada por Ribeiro et al., 2012 como la de mayor frecuencia y concentración en fluido folicular, esta banda de proteína ha sido descrita igualmente en semen bovino (Oberst et al., 2002) y en fluido uterino (Alavi et al., 2006). Principal proteína del plasma, la cual es de fundamental importancia para la regulación de la presión oncótica, transporte de hormonas tiroideas, ácidos grasos, ácidos grasos libres, mantenimiento $\mathrm{pH}$ entre otras (Fahiminiya et al., 2011;Ambekar et al., 2013). Su concentración y presencia en los perfiles podría explicar su expresión en este estudio. La banda de $57 \mathrm{KDa}$ identificada en nuestro estudio puede corresponder bien sea a la serpina o a la cadena pesada de la inmunoglobulina G (IgG) Ulbrich et al., 2009. La banda de $26 \mathrm{KDa}$ posiblemente corresponda a la proteína ligante de IGF-4 (IGFBF-4) según Gradela et al., (1998) y ha sido detectada en células de la teca, especialmente aislada de folículos de menor tamaño, dicha proteína aumenta su concentración dependiendo de los niveles de LH (Amstrong et al.,1996).

De las 25 bandas detectadas solamente la banda de 164 kDa presentó diferencias significativas $(p<0,05)$ en los diferentes tamaños del ovario derecho e izquierdo. Esta banda puede ser la descrita por Andersen et al., 1976, como una proteína del grupo de las inmunoglobulinas o de la inhibina subunidad $\alpha$ (Austin et al., 2001).

Como se describió anteriormente se encontraron correlaciones de las bandas de $164 \mathrm{kDa}$ con oocitos de calidad regular y la banda $57 \mathrm{kDa}$ con oocitos de mala calidad en los folículos $<3 \mathrm{~mm}$, al respecto Tao et al., 2014 reportaron una proteina de peso molecular aproximado 54,9 kDa como la vitamina D, la cual se asocia con atresia folicular durante la foliculogénesis (Tao et al., 2014), lo cual explicaria el resultado encontrado en la presente investigación. La banda de proteina 164 kDa se ha relacionado con inhibinas con acción principal en la secresión FSH y en otros factores producidos localmente en la gónada (IGF-I; TGF- $\beta$, activina) (Colazo y Mapletoft, 2014).

Las bandas de 26 y $38 \mathrm{kDa}$ que correlacionaron con oocitos de buena calidad en folículos de 3-6 mm pueden corresponder a proteínas identificadas como factores de crecimiento producidos en los oocitos, células tecales y de la granulosa en las diferentes etapas de desarrollo folicular, estas están involucrados en la proliferación y diferenciación de células foliculares mediante la activación de acciones de las gonadotrofinas que ejercen funciones como la esteroidogénesis, maduración del oocito (Knight y Glister 2003). El anterior argumento explicaría los resultados de este trabajo. Llama la atención que en folículos $>6 \mathrm{~mm}$ la acción de la banda de $26 \mathrm{kDa}$ tiene un efecto contrario al mencionado anteriormente con respecto a la calidad del oocito.

En el caso de la evaluación de calidad por cúmulos y citoplasma del oocito (Solís et al., 2012), los oocitos calidad tipo I evaluados en nuestros estudios presentan porcentajes inferiores a los reportados en vacas y novillas (Rizos et al., 2005); y en ovarios de hembras Bubalus bubalis utilizando la técnica de disección foli- 
cular (Quintana et al., 2012), pero fueron similares a los porcentajes descritos por Leal et al., 2010 en esta misma especie.

El número de oocitos calidad grado Gll fue mayor en folículos con tamaños $<3$ y $6 \mathrm{~mm}$, del ovario derecho. En este mismo tamaño de folículos, el porcentaje de oocitos clasificados como calidad GIII es mayor $(p<0.05)$ en el ovario izquierdo. Los porcentajes reportados en el Grado II de calidad oocitaria son ligeramente inferiores a los obtenidos en búfalos en técnicas de aspiración y disección de folículos (Leal et al., 2010; Quintana et al., 2012) y en aspiración guiada por ultrasonido en vacas y novillas (Rizos et al., 2005).

Este resultado sugiere existen diferencias entre los ovarios, asociadas con los oocitos de calidad mala (GIII) y el tamaño folicular clasificado como II, debido al fase de crecimiento del folículo en el ciclo estral. Por otra parte, al considerar los valores obtenidos por tamaño de folículos I, II, III y calidad buena (GI) y regular GII de los oocitos, su porcentaje es de $51 \%$ oocitos similar a los que se seleccionan para procesos in vitro.

\section{Conclusiones}

La concentración de proteína total en el fluido folicular es un indicador de la calidad del oocito.

Este trabajo permitió establecer la presencia de determinadas bandas de proteínas en etapas específicas del desarrollo folicular.

Los resultados de esta investigación demostraron la importancia del análisis proteómico en la búsqueda de bandas de proteínas asociadas en la calidad del oocito.

La expresión, presentación y concentración de bandas de proteína varían con el tamaño del folículo, y la composición del fluido influye en la calidad del oocito.

\section{Recomendaciones}

Los resultados encontrados ameritan realizar nuevas investigaciones que permitan aclarar la función de estas proteínas en el crecimiento folicular.

Es conveniente adelantar procedimientos más sensibles que permitan obtener información de las diferentes etapas del ciclo estral en bovinos y realizar la secuenciación las proteínas detectadas.

A partir de esta investigación se pueden adelantar estudios en búsqueda de marcadores de calidad oocitaria que permitan seleccionar hembras de mayor fertilidad.

\section{Agradecimientos}

Corporación Colombiana de Investigación Agropecuaria. AGROSAVIA

Frigorífico-Friogan Villavicencio

Dra. Shirley Flórez.

\section{Referencias}

Alavi-Shoushtari S, Asri-Rezai S, Abshenas J. A study of the uterine protein variations during the estrus cycle in the cow: A comparison with the serum proteins. Anim Reprod Sci, 2006;96(12):10-20

Aller JF, Callejas SS, Alberio RH. Biochemical and steroid concentrations in follicular fluid and blood plasma in different follicular waves of the estrous cycle from normal and superovulated beef cows. Anim Reprod Sci, 2013;142:113-120

Ambekar A, Nirujogi R, Srikanth S, Chavan S, Kelkar D, Hinduja I, et al. Proteomic analysis of human follicular fluid: A new perspective towards understanding folliculogenesis. J Proteomics. 2013;87:68-77

Andersen M, Kroll J, Byskov A, Faber M. Protein composition in the fluid of individual bovine follicles. J Reprod Fertil. 1976;48(1):109-118

Angelucci S, Ciavardelli D, Di Giuseppe F, Eleuterio E, Sulpizio M, Tiboni GM, et al. Proteome analysis of human follicular fluid. Biochim Biophys Acta - Proteins Proteomics. 2006;1764(11):1775-1785

Armstrong D, Hogg C, Campbell B, Webb R. Insulin-like growth factor (IGF)-binding protein production by primary cultures of ovine granulosa and theca cells. The effects of IGF-I, gonadotropin, and follicle size. Biol Reprod. 1996;55(5):1163-1171.

Austin EJ, Mihm M, Evans ACO, et al. Alterations in Intrafollicular Regulatory Factors and Apoptosis During Selection of Follicles in the First Follicular Wave of the Bovine Estrous Cycle. Biol Reprod. 2001;64:839-848

Bianchi L, Gagliardi A, Campanella G, Landi C, Capaldo A, Carleo A, et al. A methodological and functional proteomic approach of human follicular fluid en route for oocyte. J Proteom. 2013;90:61-76

Bijttebier J, Tilleman K, et al. Comparative proteome analysis of porcine follicular fluid and serum reveals that excessive alpha (2)-macroglobulin in serum hampers successful expansion of cumulus-oocyte complexes. Proteomics, 2009;9:4554- 4565

Bradford MM. A rapid and sensitive method for the quantitation of microgram quantities of protein utilizing the principle of protein-dye binding. Anal Biochem. 1976;72:248-254

Briggs D, Sharp D, Miller D, Gosden R. Transferrin in the developing ovarian follicle: evidence for de-novo expression by granulosa cells. Mol Hum Reprod; 1999;5(12):1107-1114

Clarke H, Hope S, Byers S, Rodgers RJ. Formation of ovarian follicular fluid may be due to the osmotic potential of large glycosaminoglycans and proteoglycans. Reprod. 2006;132(1):119-131 
Colazo MG, Mapletoft RJ. Fisiologia del ciclo estral bovino. Conference Paper. De Jong G, van Dijk JP, van Eijk HG. 1990. The biology of transferrin. Clin Chim Acta. 2014;190(1-2):1-46

Dekel N. Cellular Biochemical and molecular mechanisms regulating oocyte maturation. Mol cell endocrinol. 2005;235:19-25

Driancourt MA. Regulation of ovarian follicular dynamics in farm animals. Implications for manipulation of reproduction. Theriogenology. 2001;55(6):1211-1239

Dumont J, Umbhauer $\mathrm{M}$, et al. p90Rsk is not involved in cytostatic factor arrest in mouse oocytes. J Cell Biol. 2005;169:227-231

Fahiminiya S, Reynaud K, Labas V, Batard S, Chastant-Maillard S, Gérard N. Steroid hormones content and proteomic analysis of canine follicular fluid during the preovulatory period. Reprod Biol. 2010;8(1):132

Fahiminiya S, Labas V, Roche S, Dacheux J, Gérard N. Proteomic analysis of mare follicular fluid during late follicle development. Proteome Sci. 2011;9:54.

Fortune JE, Hansel W. Concentrations of steroids andgonadotroipns in follicular fluid from normal heifers and heifersprimed for superovulation. Biol Reprod. 1985;32:1069-1079

Fu Q, Huang Y, Wang Z, Chen F, Huang D, Lu Y, et al. Proteome Profile and Quantitative Proteomic Analysis of Buffalo (Bubalusbubalis) Follicular Fluid during Follicle Development. Int J Mol Sci. 2016;17(5):618. Doi: 10.3390/ijms17050618

Gérard N, Loiseau S, Duchamp G, Seguin F. Analysis of the variations of follicular fluid composition during follicular growth and maturation in the mare using proton nuclear magnetic resonance (1H NMR). Reprod. 2002;124(2):241-248

Gradela A, Roncoletta M, Morani C, Esper C, Franceschini P. Proteínas ligantes do insulin-like growth factor (IGFBPs) e dominância folicular em vacas Bos taurus indicus puras e cruzadas. Braz J Vet Res Anim Sci. 1998;35(5):218-220

Hill D J, Growth factors and the ircellular actions. J Reprod Fertil. 1989;85:723-734

Jans D, Hassan G, Nuclear targeting by growth factors, cytokines and their receptors: a role insignaling?. Bioessays. 1998;20:400411

Knight $\mathrm{P}$, Glister $\mathrm{C}$. Local roles of TGF- $\beta$ superfamily members in the control of ovarian follicle development. Anim Reprod Sci. 2003;78:165-183

Kubelka M, Motlik J, et al. Butyrolactone I reversibly inhibits meiotic maturation of bovine oocytes, without influencing chromosome condensation activity. Biol Reprod. 2000;62:292-302

Laemmli UK. Cleavage of structural proteins during the assembly of the head of bacteriophage T4." Nature. 1970;227(259):680685

Leal LS, Moya CF, Fernandes CB, Martins LR, Landim FC, Oba E. 2010. Evaluation of recovery, quality and in vitro nuclear maturation of oocytes obtained from Buffalo and Bovine Ovaries. 9 no Congreso de búfalo, Argentina.
Leroy J, Vanholder T, Delanghe J, Opsomer G, Van Soom A, Bols P, De Kruif A. Metabolite and ionic composition of follicular fluid from different-sized follicles and their relationship to serum concentrations in dairy cows. Anim Reprod Sci. 2004; 80(34):201-211

Monget P, Besnard N, Huet C, Pisselet C, Monniaux D. Insulin-like growth factor-binding proteins and ovarian folliculogenesis. Horm Res. 1996;45(3-5):211-217

Mortarino M, Vigo D, et al. Two-dimensional polyacrylamide gel electrophoresis map of bovine ovarian fluid proteins. Electrophoresis. 1999;20:866-869

Nandi S, Girish-Kumar V, Manjunatha BM, Gupta PSP. Biochem-ical composition of ovine follicular fluid in relation to follicle size. Dev.Growth Differ. 2007;49:61-66

Oberst E, Jobim M, Cimarosti H, Souza D, Salbego C, Wald V, Mattos R. Imunoidentificação de Albumina e Osteopontina no Plasma Seminal de Reprodutores Taurinos e Zebuínos. Semina: Ciências Agrárias, 2002;23(1):21-28

Otsuka F. Multiple endocrine regulation by bone morphogenetic protein system. Endocr J. 2010;57(1):3-14

Petrucci BPL, Wolf CA, Arlas TR, Santos GO, Estanislau JF, Fiala S, Mattos RC. Proteomics of mare follicular fluid during follicle development. J Equine Vet Sci. 2014;34(1):115-116

Psilopanagioti A, Papadaki H, Kranioti EF, Alexandrides TK, Varakis $\mathrm{JN}$. Expression of adiponectin and adiponectin receptors in human pituitary gland and brain. Neuroendocrinology; 2009;1:3847. doi: 10.1159/000151396

Quintana MD, Campos PEC, Herrera P, Gallego C, Padrón E. Comparación de dos métodos de recolección de ovocitos inmaduros para fertilización in vitro FIV obtenidos de hembras bubalus bubalis enviadas a matadero. Rev Salud Anim. 2012;34(1):53-56

Rahman Zia-Ur, Bukhari SA, Ahmad N, Akhtar N, ljaz A, Yousaf MS, Haq IU. Dynamics of follicular fluid in one-humped camel (Camelus dromedarius). Reprod Domes Anim. 2008;43:664-671

Ribeiro R, Santos A, Castilho C, Giometti J, Guaberto L, Ambiel A, Giometti I. Perfil proteico do líquido folicular coletado de ovários em diferentes fases do ciclo estral de bovinos. Colloquium Agrariae. 2012;8(2):65-74

Rizos D, Burke L, Duffy P, Wade M, Mee JF, Farrel KO, et al. Comparisons between nulliparous heifers and cows as oocyte donors for embryo production in vitro. Theriogenology. 2005;63:939-949

Rodriguez F, Martinez A, Tovar S, Pinilla L, Tena-Sempere M, Dieguez $\mathrm{C}$, et al. Regulation of Pituitary Cell Function by Adiponectin. Endocrinology. 2007;148(1):401-410

Shabankared HK, Kor NM, Hajarian $\mathrm{H}$. The influence of the corpus luteum on metabolites composition of follicular fluid from different sized follicles and their relationship to serum concentrations in dairy cows. Anim Reprod Sci. 2013;140:109-114

Shamay A, Homans R, Fuerman Y, Levin I, Barash H, Silanikove N, et al. Expression of Albumin in Nonhepatic Tissues and its Synthe- 
sis by the Bovine Mammary Gland. J Dairy Sci. 2005;88(2):569576

Schweigert FJ, Gericke B, Wolfram W, Kaisers U, Dudenhausen JW. Peptide and protein profiles in serum and follicular fluid of women undergoing IVF. Human Reproduction. 2006;21(11):29602968

Sirard MA, Richard F, et al. Controlling meiotic resumption in bovine oocytes: a review. Theriogenology. 1998;49:483-497

Solís CA, Guerra R, Sandoya G, De Armas R. Efecto de sincronización de la onda folicular y de la frecuencia de aspiración de folículos en novillas de la raza Brahman. REDVET Rev Electrón Vet. 2012;13(10):1-16

Sousa PA, Silva SJM, et al. Neurotrophin signaling in oocyte survival and developmental competence: A paradigm for cellular totipotency. Cloning Stem Cells. 2004;6:375-385

Stojkovic M, Machado SA, et al. Mitochondrial distribution and adenosine triphosphate content of bovine oocytes before and after in vitro maturation: correlation with morphological criteria and developmental capacity after in vitro fertilization and culture. Biol Reprod. 2001;64:904-909

Sunderland SJ, Knight PG, et al. Alterations in Intrafollicular Levels of Different Molecular-Mass Forms of Inhibin During Development of Follicular-Phase and Luteal-Phase Dominant Follicles in Heifers Biol of Reprod 1996;54:453-462

Tabatabaei S, Mamoei M, Aghaei A. Dynamics of ovarian follicularfluid in cattle. Comp Clin Pathol. 2011;20:591-595

Tao J, Zhao G, Zhao X, Li F, Wu X, Hu J, Zhang Y. Proteomic analysis of the follicular fluid of Tianzhu white yak during diestrus. Int J Mol Sci. 2014;15(3):4481-4491
Ulbrich S, Frohlich T, Schulke K, Englberger E, Waldschmitt N, Arnold G, et al. Evidence for Estrogen-Dependent Uterine Serpin (SERPINA14) Expression During Estrus in the Bovine Endometrial Glandular Epithelium and Lumen. Biol Reprod. 2009;81(4):795805

Valck SDM, De Bie J, Michiels ED, Goovaerts IG, Punjabi U, RamosIbeas $\mathrm{P}$, Leroy JL. The effect of human follicular fluid on bovine oocyte developmental competence and embryo quality. Reproductive Bio Medicine Online. 2015;30(2): 203-207

Villa N, Pulgarín E, Tabares P, Angarita E, Ceballos A. Medidas corporales y concentración sérica y folicular de lípidos y glucosa en vacas Brahman fértiles y subfértiles. Pesqui Agropecu Bras. 2009;44(9):1198-1204

Waki H, Yamauchi T, Kamon J, Ito Y, Uchida S, Kita S, et al. Impaired multimerization of human adiponectin mutants associated with diabetes. Molecular structure and multimer formation of adiponectin. J Biol Chem. 2003;278(41):40352-40363

Wang W, Day B N, et al., How does polyspermy happen in mammalian oocytes Microsc Res Tech. 2003;61:335-341

Yoo S, Bolbot T, Koulova A, Sneeringer R, Humm K, Dagon Y, et al. Complement factors are secreted in human follicular fluid by granulosa cells and are possible oocyte maturation factors. J Obstet Gynaecol Res. 2013;39(2):522-527

Zachut M, Sood P, Levin Y, Moallem U. Proteomic analysis of preovulatory follicular fluid reveals differentially abundant proteins in less fertile dairy cows. J Proteomics. 2016;139:122-129
Eliana Neira:https://orcid.org/0000-0002-8940-5885 José Velásquez:https://orcid.org/0000-0001-8023-1367 Jaime Cardozo:https://orcid.org/0000-0002-7744-2881 Agustín Gongora:https://orcid.org/0000-0002-2915-683X Sonia Gutierrez:https://orcid.org/0000-0002-8329-8302 Lidy Castillo: https://orcid.org/0000-0002-9437-0728 